\title{
Option
}

\section{Brevetabilité du vivant : du biobrevet au bio-big-bang}

\author{
Jean Semal \\ Résidence L'échiquier, \\ 6, Boulevard Dolez, \\ Boîte B41, \\ 7000 Mons \\ Belgique \\ <echiquier.jeansemal@euphonynet.be>
}

\begin{abstract}
Résumé
Depuis 1980, de nombreux brevets ont été concédés pour protéger la propriété intellectuelle sur les objets vivants. En conséquence, certaines situations conflictuelles ont été créées en matière d'innovation, d'application ou d'infraction. Quatre cas spécifiques ont été analysés à cet égard : i) le séquençage du génome humain où la pression politique et éthique a contenu l'effet des brevets par la publication généralisée des résultats; ii) le procès Monsanto contre Schmeiser, accusé d'infraction à la législation sur les brevets, qui aboutit à un partage d'opinion des juges de la Cour suprême du Canada ; iii) le brevet accordé par l'Office américain des marques et brevets à la variété de haricot jaune "Enola ", qui fut révoqué après six années de procédure administrative; iv) le brevet européen protégeant un produit fongicide extrait à partir de graines de margousier, qui fut révoqué après dix années de procédure. En conclusion, il apparaît que la protection de la propriété intellectuelle d'objets vivants devrait s'articuler avec le concept de "bionomie " qui traite de la gouvernance de la biosphère. En matière bionomique, il y aurait lieu à cet égard d'améliorer la cohérence opérationnelle et légale de la protection des droits relatifs aux objets biologiques. S'agissant des itinéraires techniques agricoles, il s'agit de résoudre correctement l'équation qui relie les gènes, les organismes et les cultures dans les systèmes de production et de valorisation.
\end{abstract}

Mots clés : biopiratage ; brevetabilité ; organisme vivant ; révocation.

Thèmes : méthodes et outils ; productions végétales ; productions animales.

\section{Abstract \\ Patentability of living organisms: From biopatent to bio-big-bang}

Inventions, like processes or products, have long been protected in many ways: secrecy, monopoly, trademarks, international conventions, regulations by public authorities. Nowadays, intellectual property rights (IPR) are regulated under the "Trips" system (Trade-Related Intellectual Property Rights) within the World Trade Organisation (WTO). WTO members have to protect innovative products or processes either by awarding a utility patent or through sui generis systems. For decades, plant breeders have developed "plant variety protection certificates" which are awarded under an international UPOV convention implemented in 1968, revised in 1990. Since the 1980 decision of the US Supreme Court, living organisms or part thereof (including genes) became patentable subject matter. Thereafter, large numbers of patents have been delivered to life forms, some of which resulting in conflicting situations in terms of novelty, applicability, or infringment of biopatents. To illustrate some of the situations involved, four specific cases are analysed in this article, in order to evaluate the limits of biopatentability: i) Sequencing the human genome. The improvement of DNA sequencing techniques paved the way for characterizing the entire human genome. In the early stages of the project, results were published. In 1988, however, patents were requested for "Est", i.e. expressed sequence tags whose location or function were unknown. After a period of refusal, such patents were granted in the USA and later on in Europe, notwithstanding the opposition of a fraction of scientists who disputed their status of "novelty", considering it was like unduely patenting knowledge. After a period of unrest, President Clinton and Prime Minister Blair called for the publication of the whole genome sequence to the benefit of mankind. This was done, thus precluding further patentability of the human genome; ii) The saga of Monsanto suing the farmer Schmeiser. Percy Schmeiser successfully cropped canola in Saskatchewan (Western Canada) during half a century. Tests conducted in the 1998 crop revealed a high resistance to the glyphosate-based weed killer Roundup, due to the prevalence of genes patented by Monsanto and Monsanto Canada Inc. As Schmeiser never purchased Roundup-resistant canola seeds, nor obtained a licence to use them, Monsanto brought an 
action for patent infringement. Schmeiser denied any intentional introduction of patented genes on his land, and accused Monsanto of inadequate confinement of its patented material. The trial judge found the patent to be valid and allowed the action, granting \$CAN 19,832 compensation to Monsanto, together with \$CAN 153,000 of judiciary costs. The Federal Court of Appeal affirmed the decision and Schmeiser brought the appeal to the Supreme Court. The Court split five to four on the matter. The five majority Justices confirmed that the farmer was guilty of infringement for "using" patented canola without due authorization. Compensations were denied to Monsanto, however, because the company had elected accounting of profits as remedy. Considering that the appellant earned no profit from the invention, the Court ruled that the respondents were entitled to nothing on their claim of account. In view of this mixed result, it resolved that each party bear its own costs throughout. The four minority Justices expressed views opposed to the majority ruling. They considered that plants, or their offspring, were unpatentable by law, so that a person skilled in the art could not be expected to realize that patent protection of genes extends to standing crops. They therefore estimated that the lower Courts erred in considering gene patent infringement as applicable to plants and harvested grains. Furthermore, the complexity and nuances of "innocent bystander" protection, in the context of agricultural biotechnology, should be expressly considered by Parliament, because it can only be inadequately accommodated by the law in use; iii) Revocation of the US patent granted to the yellow bean variety Enola. In 1999, the US Patent and Trademark Office (USPTO) patented a variety of common bean with yellow-coated seeds, under the name Enola. Originally, Enola parents had been bought on a market place in Mexico. After selection in Colorado State, Enola was patented. As yellow-seed beans are much appreciated by latinos, both in Mexico and in the United States, patenting Enola was challenged by Mexican bean growers, who argued the lack of novelty compared to yellow bean varieties previously cropped in their country. After a legal battle and further scientific evidence based on DNA comparison, it appeared that Enola could not be distinguished from hybrid yellow beans which preexisted in Mexico. In 2005, Enola patent was revocated for imprecision of the original request for patenting, and undue heterogeneity of parent and progeny seeds; iv) Revocation of the European patent granted to a process for the preparation of a fungicide from neem extracts. In 1994, the European Patent Office (EPO) patented a fungicide fraction extracted from neem, a tree originating from the Indian peninsula. Neem seed extracts had been used for many centuries in India, mainly as medicine, cosmetics, and control agents against fungi or insects. The patent, which had been granted jointly to Grace $\mathrm{C}^{\circ}$ (New York) and to the US Department of Agriculture (USDA), was challenged by a consortium of three ladies: a Belgian representative to the European parliament, together with an Indian NGO activist and an American NGO activist. In 2000, EPO revocated the patent for lack of inventivity, but the US government, together with Grace $C^{\circ}$, called for appeal. Five years later, on march 8, 2005, the patent was made irrevocably void. The appeal body of EPO stated that patenting was denied to items previously known, including through local tradition. It should be noted that, during ten years, the neem marked had been in trouble as the result of the patent, thus inflicting indirect damage to the traditional users of neem extracts. It is concluded that patentability of living items should be clarified by including protection of intellectual property rights within the concept of "bionomy", which covers the highly complex components interacting in biosphere governance. The mere application of regular patent rules to biological items in a climate of "commoditization", resulted in undue patent delivery, which had either to be revocated (yellow-seed bean variety, fungicidal neem extracts), or to be retracted under political and ethical pressure (sequencing the human genome). Patent infrigment in open-field agriculture revealed very complex interactions, with split evaluation by judges in Court. Judiciarisation of this sector should receive due attention, in order to improve the coherence, both operational and legal, in protecting the mixed property rights involved. Time has come to solve the equation expressing relationships between these property rights, when applied to genes, organisms, or crops within the complexity of open-field agricultural processes.

Key words: biopiracy; living organisms; patentability; revocation.

Subjects: tools and methods; vegetal productions; animal productions. 
appropriation privée des biens de la nature et des réalisations inventives du génie humain s'est concrétisée de longue date sous les formes les plus diverses du fait et du droit. Parmi les principales modalités observées, on peut distinguer le secret de fabrication, le monopole, la convention, ou encore la régulation par la puissance publique, notamment sous forme de droits d'auteur, de marque ou de label protégés, d'appellations plus ou moins contrôlées.

Le monopole territorial est encore largement opérationnel de nos jours, notamment en matière pétrolière ou gazière (l'actualité l'illustre régulièrement), de gestion des bassins hydrologiques, ou encore de zones de pêche.

S'agissant des organismes vivants, plusieurs cas emblématiques illustrent des situations de monopole de fait, dont certains durèrent pendant de longues périodes, mais qui furent tous contournés par des voyageurs audacieux bravant les interdits locaux, parfois au péril de leur vie. Ce fut le cas notamment du café, du ver à soie et de l'hévéa (Semal, 2006), mais aussi de diverses épices, dont la noix de muscade.

La saga du café commence dans les Contes des Mille et Une Nuits, lorsque Shéhérazade raconte l'histoire d'un jeune berger qui avait constaté que ses chèvres veillaient la nuit lorsqu'elles avaient brouté un arbuste particulier. Il vint confier son observation à des moines soufi qui virent tout le profit à tirer de cette plante pour accroître leurs périodes de veille permettant la prière. La nouvelle se répandit dans la région, qualifiée à l'époque d' "Arabie heureuse " et les cultures de café proliférèrent au Yémen. L'Occident eut vent de la chose et la caféomanie conquit l'Europe via les Ottomans et Venise. Â partir du XvI siècle, le port de Mokka, sur la mer Rouge, se spécialisa dans l'exportation de la précieuse denrée. Pour conserver le monopole de leur production caféière, les Yéménites auraient traité leurs graines de café par la chaleur et certains y voient une des origines de l'actuelle torréfaction. L'Arménien Pascal ouvrit le premier "café " de Paris, suivi du Sicilien Procope Colteni qui accrocha son enseigne rue de l'Ancienne Comédie. Le "Procope" fait toujours le bonheur des amateurs de bonne chère.

Le monopole arabe prit fin lorsqu'un pèlerin revint de La Mecque en cachant sept graines de café vert dans la doublure de son pourpoint. En 1640, le café colonisait l'île de Java, puis après un crochet par Amsterdam et Paris, fut transféré aux Amériques par le capitaine de Clieu en 1723.

Le ver à soie (Bombyx mori) est originaire de Chine, mais la provenance, ainsi que les modalités de fabrication de la soie, demeurèrent mystérieuses en Occident pendant des siècles. Seules les caravanes amenant le précieux tissu par les routes de la soie faisaient le lien avec l'Orient. Le monopole asiatique fut rompu en 552 par deux religieux qui rapportèrent des cocons dissimulés dans une canne de bambou et les offrirent à l'empereur byzantin Justinien. De Constantinople, le ver à soie se répandit dans toute l'Europe où il assura la prospérité de maintes régions.

Quant au caoutchouc, qui alimenta la fabrication des pneumatiques à partir de la fin du $\mathrm{XIX}^{\mathrm{e}}$ siècle, il fit l'objet d'un quasi-monopole du Brésil et du Congo jusqu'à ce qu'un explorateur anglais subtilise des graines d'hévéa. Après un bref passage à Kew Gardens, on transféra les précieuses plantules à Ceylan et en Malaisie. Bientôt, l'Asie produisit davantage de latex que l'Amazonie.

La rupture de monopole existe toujours à l'ère de la biologie moléculaire et un cas vécu en témoigne. En 1970, un chercheur écrit à un collègue pour qu'il lui procure un bactériophage décrit dans une publication récente. Par retour du courrier, son correspondant l'informe que le phage n'est plus disponible. Aussitôt, le quémandeur éconduit découpe l'enveloppe et place les morceaux sur un milieu de culture. Quelques jours plus tard, il isolait le phage convoité. Aujourd'hui, le courrier électronique, bien que chargé en virus, ne permettrait plus une telle prouesse.

\section{La protection de la propriété intellectuelle}

L'historique et l'évaluation des modes de protection de la propriété intellectuelle des organismes vivants ont fait l'objet d'excellentes revues de synthèse (Feyt, 2001a ; Feyt, 2001b ; Feyt, 2004 ; Gepts, 2004). La question relève désormais de l'Organisation mondiale du commerce (OMC) héritière du GATT (General
Agreement on Tariffs and Trade) au sein duquel ont été conclus les accords Trips (Trade-related Intellectual Property Rights). Ces accords prévoient que les États s'engagent à établir des systèmes de brevet ou de protection sui generis des plantes et des animaux, le brevet étant obligatoire pour les micro-organismes.

Le brevet est un privilège de protection temporaire accordé aux inventeurs qui acceptent de divulguer leur savoir-faire au profit de tous. Le Congrès des ÉtatsUnis en 1790 et l'Assemblée constituante française en 1791 en ont formulé les règles sous forme d'un contrat entre un inventeur et la puissance publique, afin que l'invention décrite par l'auteur soit exploitée par des tiers moyennant paiement d'une redevance. Sans valoir titre de propriété, ce privilège était justifié par la stimulation attendue des applications techniques et de la commercialisation de leurs produits.

Dans un premier temps, les organismes vivants ne sont pas brevetables, n'étant pas considérés comme "invention". Cependant, Pasteur obtient un brevet en France en 1865 et aux États-Unis en 1873 pour une souche de levure dépourvue de contaminations bactériennes. Aux ÉtatsUnis, des variétés de poires et de pommes ont été brevetées dans les années 1930, mais il faudra attendre 1980 pour que la Cour suprême de ce pays décide, par 5 voix contre 4 , de breveter une bactérie transgénique capable de dépolluer les sols souillés par le pétrole. Cette décision mettait fin à un litige de 8 années entre la société requérant le brevet et l'Office des brevets qui le lui refusait, en prétextant que les organismes vivants n'étaient pas brevetables.

S'en suivirent des brevets accordés pour des huîtres en 1987 et à une souris sensible aux cancers en 1988. En Europe, la directive de 1998 sur la brevetabilité du vivant confie à l'Office européen des brevets (OEB) le droit de breveter des plantes et des animaux pour autant qu'elles soient suffisamment innovantes et susceptibles d'applications commercialisables. Les variétés ne sont pas brevetables en tant que telles, mais peuvent être brevetées si leur génome a été modifié par transgenèse. Toute cellule contenant le gène breveté, de même que les plantes qui en sont issues, est couverte par le brevet.

S'agissant de variétés végétales, la protection des droits des obtenteurs de nouvelles variétés a fait l'objet d'un accord international entre 5 pays européens créant 
l'Union pour la protection des obtentions végétales (Upov) qui entra en vigueur en 1968 et qui sera révisé en 1991. L'Upov décerne un certificat d'obtention végétale (COV) à une variété nouvelle non commercialisée répondant aux critères "DHS ", à savoir Différente (par rapport aux variétés existantes), Homogène, et Stable (reproductible à l'identique).

La variété nouvelle couverte par un COV ne peut être copiée, mais elle peut être utilisée librement comme géniteur dans la création d'une nouvelle variété, suffisamment distincte du parent protégé. Cela indique que c'est le talent d'assembleur de gènes de l'améliorateur qui est reconnu et protégé par le système Upov et non les gènes eux-mêmes, comme dans le brevet.

L'Upov, qui rassemblait 49 pays adhérents en 2001, a permis de développer avec succès de nouvelles variétés végétales et constitue aujourd'hui un système performant permettant de répondre aux exigences de l'OMC.

La convention internationale sur la biodiversité de 1992 a introduit de nouveaux paramètres qui intéressent surtout les pays en développement. Il s'agit notamment du privilège de l'agriculteur, qui peut ressemer une variété protégée qu'il a lui-même produite sur son exploitation. Il s'agit également de protéger les droits des communautés rurales qui ont amélioré, au cours du temps, le potentiel génétique de leurs productions par des méthodes traditionnelles appropriées aux cultures de subsistance, ce qui crée des situations d'antériorité en matière d'attribution de brevets.

L'ensemble de cette problématique est source de conflits entre États, entre groupes d'intérêts: agriculteurs, améliorateurs, consommateurs, firmes de biotechnologie, pays industrialisés versus pays pauvres, groupes de pression favorables ou hostiles à la brevetabilité des organismes vivants.

Sur le proscenium, ces divergences se traduisent par des joutes politicomédiatiques et par des échanges d'argumentaires scientifico-philosophico-idéologiques. Cependant, dans les coulisses, se déroulent des tractations économicofinancières où s'affrontent les différents lobbies des opérateurs concernés. La judiciarisation croissante des rapports sociétaux se traduit par des actions de plus en plus nombreuses devant les instances judiciaires ou administratives. Nombre d'entre elles n'aboutissent pas, soit que les plaignants soient incapables de sup- porter les charges des procédures, soit qu'ils se désistent moyennant compensations financières. De cette manière, des pans entiers de la réalité échappent à l'analyse objective.

Occasionnellement cependant, les procédures engagées vont à leur terme décisionnel, ce qui permet d'expliciter les modes opératoires, ainsi que les arguments et prétentions des parties, sur la base de documents officiels et d'établir des jurisprudences dans des matières nouvelles qui en sont quasi démunies.

Dans le présent article, quatre cas ont été retenus, explicitant des situations emblématiques:

- le "génome humain" qui fut un des détonateurs de la boulimie biobrevetante : - le procès opposant la multinationale Monsanto à l'agriculteur Percy Schmeiser ; - l'abrogation d'un brevet américain attribué à une variété de haricot ;

- l'abrogation d'un brevet européen accordé à un fongicide extrait du margousier.

\section{Quand les biobrevets deviennent boulimiques}

Le premier exemple traité concerne le programme Hugo (Human genome) qui a conduit à des arbitrages politiques au plus haut niveau. Le second cas se rapporte à l'interminable procès intenté par la firme Monsanto contre l'agriculteur Percy Schmeiser, qui aboutit à la division des juges de la Cour suprême du Canada dans le prononcé du jugement final de la cause.

\section{Le génome humain : sauvé ou manipulable?}

Les perspectives de décryptage du génome humain et les espérances de valorisation socio-économique d'une telle avancée scientifique ont occupé les esprits dans la dernière décennie du $\mathrm{Xx}^{\mathrm{e}}$ siècle. Il y fut fait allusion dans une brève des Cabiers Agricultures (Semal, 2005a). La question a fait couler beaucoup d'encre, de salive et de pixels (Semal, 2006 ; Sulton, 2004). Le décryptage du génome de virus, puis de bactéries, de champignons et d'organismes végétaux et animaux, avait montré les potentialités des techniques de séquençage et enthousiasmé les chercheurs. L'identification de gènes humains liés à des pathologies, à des risques, à des performances ou à des comportements particuliers, avait mis en appétit les opérateurs techno-scientifiques, médicaux, sociopolitiques et médiatiques. L'idée d'un décryptage de l'entièreté du génome humain fut débattue pour la première fois en public en 1985 , lors d'un congrès tenu à Santa Cruz (ÉtatsUnis). À l'époque, les connaissances relatives à la structure, au positionnement et au fonctionnement des gènes humains étaient fragmentaires, tandis que les performances des techniques de séquençage apparaissaient limitées et leurs coûts prohibitifs.

Les péripéties initiales du programme ont été décrites (Semal, 2005a). En 1988, le projet Human genome prend de l'assurance aux États-Unis, avec un budget de 200 millions de dollars. Sur le plan international, on crée Hugo (Human Genome Organization) avec notamment la collaboration de la Grande-Bretagne (en la personne de Francis Collins, soutenu par le Wellcome Trust), avec Craig Venter du National Institute of Health (NIH) pour les États-Unis et avec la participation de laboratoires français (la France avait participé à la phase initiale via le Généthon), allemands, japonais et chinois.

En 1998, Craig Venter fonde la société Celera Genomics en vue d'accélérer le processus et de terminer seul le séquençage. Celera se dote de 300 séquenceurs fonctionnant en permanence, couplés à 4200 ordinateurs qui analysent les résultats. Après un an de fonctionnement, Celera, fondée avec un capital initial de 70 millions de dollars, atteint une capitalisation boursière de 1,3 milliard de dollars. Dès 1990, Venter avait introduit la notion d' "Est " (expressed sequence tags), séquences partielles de zones codantes du génome, en vue de les breveter. Des demandes de brevets pour des milliers d' "Est " sont déposées, mais dans un premier temps, les Offices compétents des États-Unis (US Patent and Trademark Office, USPTO) et d'Europe (OEB) refusent d'accorder les brevets sollicités. Bientôt cependant, leur résistance s'émousse et des brevets sont accordés, malgré les protestations d'une partie de la classe scientifique, qui considère inadéquat de breveter des structures dont on ignore le fonctionnement. On ne peut en effet accorder une protection juridique à de simples éléments de connaissance, sans contrevenir à la philosophie de base 
inhérente à la brevetabilité. Bientôt cependant, on se rend compte de l'inanité des brevets accordés aux "Est", d'autant que le nombre de gènes dont ils feraient partie diminue comme peau de chagrin. Un même gène risquerait donc de contenir des "Est " multiples, dont les brevets auraient été concédés à de nombreux propriétaires différents, ce qui rendrait inextricable l'exploitation ultérieure de l'entièreté du gène.

Dès lors, la pression en faveur du libre accès aux séquences de l'entièreté du génome humain (lequel avait été proclamé "patrimoine de l'humanité " en 1997, dans l'article 1 de la "Déclaration universelle sur le génome humain " lors de la Conférence générale de l'Unesco) reçoit des soutiens croissants. Le processus culmine le 15 mars 2000, lorsque le président Clinton et le premier Ministre Tony Blair se retrouvent côte à côte pour prôner cette option dans une déclaration commune. Un point de vue déjà défendu par le ministre français Hubert Curien dès 1991. La réaction n'allait pas tarder: le 26 juin 2000, le "brouillon " des séquences du génome humain était rendu public. Il fut publié en 2001, en partie dans Nature et en partie dans Science. L'ensemble du séquençage s'achevait en 2003.

Qu'allait-on faire de ces données? Comme Craig Venter l'avait prédit, on dut constater qu'on en était seulement à la fin du début et que pour exploiter ces connaissances, l'essentiel restait à faire. Selon le prix Nobel John Sulton (2004), qui coopéra au séquençage réalisé au Laboratoire Sanger de Cambridge, on ne connaît que très partiellement la composition des quelque 25000 à 30000 gènes de notre génome et beaucoup reste à préciser en ce qui concerne leur localisation, leur expression, leurs fonctions, leurs systèmes de régulation et de contrôle et, ce qui n'est pas le moindre, les interactions de leurs produits avec l'ensemble du métabolisme cellulaire. Ces incertitudes se sont reflétées au niveau des applications, qui tardent à se concrétiser, notamment sur le plan de la thérapie génique.

La tentative de mainmise privée sur le génome humain semble avoir fait long feu. Est-ce simple partie remise, ou prise de conscience des limites qu'il convient de fixer à la brevetabilité des génomes d'organismes vivants? Affaire à suivre...

\section{Monsanto contre Schmeiser : score 5-4}

Percy Schmeiser et son épouse, qui sont aujourd'hui septuagénaires, ont cultivé du colza avec succès pendant un demisiècle sur leurs terres de 300 hectares, situées dans l'Ouest du Canada. Pendant cette période, le colza est devenu particulièrement rentable comme source d'huile de table et d'aliments pour le bétail. Les Schmeiser avaient sélectionné, au cours du temps, un écotype local de colza particulièrement performant et, conformément à la coutume, ils ressemaient leurs propres graines année après année (Semal, 2006; Semal, 2002). En 1996 , plusieurs exploitations jouxtant leur propriété avaient introduit le colza transgénique "Roundup Ready" résistant à l'herbicide Roundup et breveté par Monsanto. En 1997, Schmeiser constatait avec étonnement que des colzas résistants au Roundup poussaient dans ses fossés désherbés. Comme il n'avait pas introduit le transgène sur ses terres, il conduisit sa culture comme à l'ordinaire et utilisa des graines de sa récolte pour les semis de 1998. En 1998, à la demande de Monsanto, des échantillons furent prélevés dans les champs des Schmeiser, qui montrèrent un taux élevé de résistance au Roundup.

L'agriculteur n'ayant pas souscrit à l'indispensable " accord d'utilisation de technologie", ni acheté de semences chez un revendeur agréé, fut assigné en justice en août 1999 par Monsanto, qui requérait une injonction lui interdisant de récolter, cultiver ou vendre des graines, cellules ou plantes de colza contenant le gène breveté.

Les arguments des parties au procès et les attendus des jugements successifs forment un corpus qui illustre les réalités de terrain et les modes opératoires respectifs de l'agriculteur et de la firme de biotechnologie.

Devant le tribunal d'instance, la thèse du plaignant Monsanto fut lumineuse de simplicité : l'accusé avait, sur ses terres, en 1998, semé des graines, récolté des plantes et vendu des récoltes dont il savait, ou aurait dû savoir, qu'elles contenaient un gène breveté, sans avoir souscrit aux règles d'utilisation du brevet.

Pour sa défense, Schmeiser, tout en reconnaissant la présence de gènes brevetés dans ses cultures en 1997 et 1998, affirma que cette situation était indépendante de sa volonté et qu'elle lui avait été imposée. Selon lui, la firme détentrice du brevet avait été incapable d'assurer le confinement du gène protégé, de sorte que celui-ci s'était retrouvé indûment dans les cultures de colza non transgéniques. En octobre 2003, il nous a confirmé, en tête-à-tête, n'avoir jamais introduit sciemment des OGM dans ses parcelles.

Le jugement d'instance rendu par le juge Andrew Mc Kay le 29 mars 2001, a confirmé la légitimité du brevet de Monsanto et a estimé que Schmeiser avait contrevenu à la législation sur les brevets. Il considéra que cette infraction existait indépendamment de l'origine des transgènes et de la manière dont ils avaient été introduits dans les cultures litigieuses. En conséquence, l'agriculteur était condamné à payer 19832 dollars canadiens de dommages au profit de Monsanto et 153000 dollars canadiens de frais de justice.

La cour fédérale d'appel, siégeant à Saskatoon, confirma les décisions du jugement d'instance.

Schmeiser fit alors appel devant la Cour suprême du Canada, laquelle accepta d'entendre les parties le 20 janvier 2004 et rendit son verdict le 21 mai 2004 (Supreme Court of Canada, 2004).

La Cour de neuf membres se divisa sur la question : cinq juges formulèrent le verdict majoritaire tandis que les quatre autres se ralliaient à un point de vue opposé, ce qui illustre la perplexité de la plus haute instance judiciaire du pays.

Le jugement reconnaît la validité du brevet de Monsanto et conclut que Schmeiser s'était mis en infraction à l'égard de la législation sur les brevets. En revanche, il réforme le jugement d'instance en matière financière en considérant que Schmeiser n'avait retiré aucun bénéfice de l'utilisation de graines transgéniques. Or, c'est ce critère de compensation qui avait été choisi par Monsanto pour son dédommagement.

Les juges minoritaires, pour leur part, ont argumenté que les plantes, en tant qu'entités vivantes, ne seraient pas brevetables et que Schmeiser n'avait pas "exploité" les gènes et cellules OGM comme défini dans le brevet accordé à Monsanto.

L'accusé Schmeiser pouvait donc raisonnablement penser que la descendance de ses propres plantes transgéniques, introduites accidentellement, n'était pas concernée par le brevet en cause. D'autant que les récoltes avaient été vendues pour la trituration et l'alimentation animale et non en tant que semences. 
Pour la minorité, un brevet doit être défini de façon prévisible par le public, ce qui n'était pas le cas en l'occurrence. En fait, le gène breveté par Monsanto fait partie d'une catégorie nouvelle, dans la mesure où l'invention brevetée produit une descendance qui la contient, sans intervention humaine. Dès lors, le gène de Monsanto, au lieu d'être breveté, devrait être protégé dans le cadre du système sui generis qui existe au Canada sous le nom de "droit des sélectionneurs de végétaux ".

Les juges de la minorité concluent par ailleurs que la complexité de la biotechnologie agricole fait que "l'infraction innocente " plaidée par Schmeiser devrait être prévue expressément par le Parlement.

Il apparaît donc que, s'agissant d'une variété végétale possédant des propriétés uniques liées à la transgenèse, le couple transgenèse-site d'intégration est susceptible d'être breveté. Cependant, un tel brevet, portant sur un événement expérimental, ne devrait pas s'appliquer à la plante en tant que telle.

Ce qui précède montre que la législation sur les brevets de plantes OGM cultivées en agriculture devrait être précisée ou modifiée pour prendre en compte la contamination accidentelle des cultures en milieu ouvert. La situation actuelle est non seulement difficile à gérer sur le plan juridique, mais elle est aussi dommageable en matière commerciale et financière. En effet, les filières agroalimentaires où coexistent des cultures OGM et nonOGM ont connu des perturbations majeures au cours des années récentes. Ces difficultés risquent de perdurer selon un rapport du Conseil national de la recherche des États-Unis.

\section{Quand les biobrevets deviennent indigestes}

Récemment, deux cas d'espèce, l'un aux États-Unis, l'autre en Europe, ont mis en évidence la légèreté avec laquelle les offices des brevets de ces zones géographiques avaient accordé certains biobrevets qui furent ensuite récusés, après des années de procédure, pour des raisons parfaitement prévisibles au moment de leur attribution.

\section{Les haricots jaunes : l'étiquette innovante}

Le 13 avril 1999, l'Office américain des brevets et des marques (USPTO) accordait le brevet $\mathrm{n}^{\circ} 5.894 .079$ à une variété de haricot dénommée "Enola " par son " inventeur". La demande avait été introduite le 15 novembre 1996 par Larry L. Proctor en vue de breveter un type de haricot à graines jaunes dont la coloration demeurait stable au travers des saisons et se conservait après croisement entre individus du type breveté. Des graines de couleur jaune sélectionnées à partir d'un sachet de graines multicolores acheté sur un marché au Mexique étaient à l'origine de cette "trouvaille". Les semences jaunes furent semées en 1994 dans le Colorado et propagées par autofécondation. Après sélection sur la base des caractéristiques du feuillage et des gousses, ainsi que des propriétés de déhiscence de ces dernières, les graines de première génération furent semées en 1995. Après autofécondation et nouvelle sélection, les graines récoltées furent utilisées pour décrire la variété Enola (United States Patent and Trademark Office, 2005).

Les graines d'Enola furent déposées auprès de l'American Type Culture Collection sous le numéro ATCC 209.549. Par ailleurs, la variété fut enregistrée sous le numéro 9.700027 auprès de l'Office de protection des variétés végétales du Département américain de l'Agriculture (United States Department of Agriculture, USDA) et présentées en vue de l'obtention d'un brevet. Le brevet, accordé en 1999, couvre toutes les parties et caractéristiques d'Enola: graines (récoltées après autofécondation, rétrocroisement ou croisement avec d'autres cultivars), pollen, feuilles, gousses, tiges, cellules.

Enola possède d'excellentes caractéristiques sous tous les aspects, y compris le rendement, les qualités culinaires et alimentaires. Les populations hispanophones émigrées aux Etats-Unis, tout comme leurs congénères du Mexique, sont très friandes de haricots à graines jaunes. Les améliorateurs mexicains avaient notamment développé des hybrides très appréciés entre des haricots locaux de type Azufrado et des haricots péruviens de type Peruano. La variété hybride est cultivée dans le nord-ouest du Mexique et est très prisée par les immigrants qui ont franchi le Rio Grande à la recherche du rêve américain.

Suite à l'attribution du brevet à Larry Proctor, les importations de haricots jau- nes semblables à Enola firent l'objet d'un prélèvement de royalties au profit de Proctor, alors que les Mexicains considéraient avoir développé eux-mêmes cet hybride. Proctor et le PTO furent dès lors accusés de biopiraterie et des actions légales furent introduites pour révoquer le brevet en cause.

La chaîne de télévision française Arte a présenté une émission sur le sujet le 15 novembre 2005. On y entend les témoignages des protagonistes ayant joué un rôle en la matière.

Pour Larry Proctor, qui fait visiter fièrement ses champs du Colorado, son bon droit ne fait aucun doute : il a créé une nouvelle variété et l'a développée à grande échelle avec un succès économique évident, sous le couvert d'un brevet valant pour l'ensemble du territoire des États-Unis. Dès lors, les importations de haricots mexicains ayant les caractéristiques d'Enola doivent acquitter les droits, au même titre que les producteurs d'Enola aux États-Unis.

Les Mexicains, au contraire, plaident pour l'abrogation du brevet accordé par l'USPTO, pour cause d'antériorité d'une variété semblable développée au Mexique.

Parmi les intervenants, Paul Gepts, qui fut chercheur à la faculté agronomique de Gembloux et est aujourd'hui établi en Californie, a joué un rôle dans la procédure de recours en effectuant des analyses comparatives entre Enola et l'hybride mexicano-péruvien, sur la base du polymorphisme des fragments d'ADN (Pallottini et al., 2004). L'auteur a également comparé la couleur du feuillage, présentée comme critère de différenciation entre les deux types (Enola et Afruzado Pimono) lors de la demande de protection d'Enola en tant que variété végétale. Les résultats publiés par l'équipe de Gepts montrent que les empreintes génétiques de Enola, tout comme la coloration de son feuillage, ne peuvent être distinguées de celles des haricots mexicains du groupe "Peruano". L'article conclut en s'interrogeant sur la base rationnelle qui a conduit à l'attribution d'un brevet " utility patent "à Enola.

Les documents officiels de l'USPTO sont peu explicites à cet égard. Le rapport des examinateurs (D. W. Robinson et M. K. Kilbal) comporte 5 pages de descriptif émanant du requérant, complété par des annexes photographiques. La couleur jaune de l'enveloppe de la graine accompagnée d'un hyle annulaire est présentée comme la caractéristique 
majeure de la variété, décrite par ailleurs comme uniforme et stable.

Après diverses péripéties juridiques, avec échange de données complémentaires entre le détenteur du brevet et l'USPTO, l'Office a promulgué le 14 avril 2005, sa décision finale rejetant les éléments constitutifs du brevet 5.894.079, en réponse à l'argumentaire finalisé du détenteur du brevet introduit le 2 juin 2004.

Dans les 24 pages de justification de la décision signée par l'examinatrice E.F. McElwain, les éléments suivants sont notamment évoqués :

- vices de forme dans différents documents versés au dossier ;

- déclarations contradictoires du détenteur du brevet quant aux modalités d'obtention de la variété Enola ;

- témoignages selon lesquels le détenteur du brevet était conscient de ce qu'Enola provenait d'une sélection parmi des gaines de Azufrado Peruano 87, alors que cet élément ne figure pas dans la demande initiale de brevet ;

- les gammes de couleur définies dans la demande de brevet initiale reposent sur des observations du requérant à la lumière naturelle et ne permettent pas de comparaison objective avec d'autres variétés. Le requérant a noté lui-même que les graines du dépôt légal d'Enola varient en couleur, taille et forme, de sorte qu'on ne peut utiliser ces critères pour distinguer Enola de variétés existant antérieurement ;

- le propriétaire du brevet considère que des études complémentaires pourraient établir l'originalité d'Enola, ce qui indique que la demande initiale n'était pas suffisamment précise ;

- les données recueillies ne permettent pas de conclure à l'homogénéité génétique ou phénotypique d'Enola. En conséquence, il sera impossible de définir des critères permettant d'établir une éventuelle transgression du brevet par des tiers ;

- le détenteur du brevet et l'examinatrice diffèrent de point de vue quant aux critères de reproductibilité de la variété Enola sur la base de caractères sélectionnables stables et uniformes ;

- certains traits d'Enola sont variables et le détenteur du brevet n'a pas précisé lesquels pourraient être suffisamment stables et uniformes pour définir la variété ; - les caractéristiques d'une invention doivent être précisées par écrit ne varietur dans la demande de brevet et cette obligation n'est pas rencontrée par un dépôt de graines hétérogènes ;
- le détenteur du brevet argumente que le sélectionneur utilise le phénotype et non le génotype comme critère opérationnel. L'examinatrice note que le phénotype est l'expression fonctionnelle des séquences génomiques de la plante.

L'ensemble du document reflète un dialogue de sourds entre praticien de la sélection et gardien de la lettre dans l'attribution d'un brevet sur la base de critères stables et homogènes, susceptibles d'être opposables à des tiers qui auraient enfreint la protection légale y afférent.

On notera que les attendus de la décision se fondent sur des critères essentiels de l'admission à la liste des variétés protégées par le système Upov, à savoir "Différent-Homogène-Stable".

On remarquera par ailleurs qu'il est à peine fait allusion à ce qui aurait permis de prévenir, dès l'origine, l'attribution d'un brevet à Enola, à savoir le défaut d'innovation par rapport à une variété préexistante. Il aura fallu 6 ans aux instances officielles pour reconnaittre leur erreur en la matière.

\section{Le margousier : brevet pour une " invention " millénaire}

Le margousier (Azadirachta indica) est un arbuste du sous-continent indien dont les graines, riches en huiles, sont utilisées traditionnellement depuis des siècles comme sources de médicaments, de cosmétiques, comme agents protecteurs contre les insectes et les microbes, et comme produits à usages divers.

Les péripéties d'attribution de brevets à des extraits de margousier (neem, en langue vernaculaire et en anglais, azaddarakth ou " arbre libre ", en persan) ont déjà été évoquées (Semal, 2005b).

De nombreux brevets ont été accordés à des extraits de neem aux États-Unis, en Europe et en Inde, notamment des préparations à base d'azadirachtine en vue de lutter contre les insectes. Le 14 septembre 1994, l'Office européen des brevets (OEB) siégeant à Munich, délivrait le brevet 436.257 conjointement à la firme W.R. Grace (New York) et au secrétaire d'État américain à l'Agriculture, suite à une demande introduite le 12 décembre 1990. Il s'agissait d'une méthode de "contrôle" des attaques fongiques par application sur les champignons d'une préparation à base d'huile hydrophobe extraite du margousier.

La firme Grace allait bientôt se heurter à l'opiniâtreté juridique de "Trois Grâces ", à savoir Magda Alvoet, alors députée européenne, actuellement ministre d'État et ex-ministre de la Santé et de l'Environnement du Gouvernement belge, agissant au nom des Verts européens, la Dr Vandana Shiva, animatrice d'une Organisation non gouvernementale (ONG) indienne (Fondation de recherche pour la science, la technologie et l'écologie) et l'Américaine Linda Bullard au nom de la Fédération internationale des mouvements d'agriculture biologique. Les opposantes s'adjoignirent les conseils du professeur Fritz Dolder, de l'université de Bâle, pour les représenter pendant les dix années que dura la procédure.

Outre des défauts de type juridique, deux arguments majeurs furent mis en avant par les plaignantes: i) l'antériorité, en Inde, d'un produit analogue pour soigner les dermatoses et pour protéger les céréales contre les attaques fongiques, ce qui mettait en question l'aspect novateur et l'activité inventive requis par la Convention sur le brevet européen (CBE) ; et ii) l'établissement d'un monopole indirect sur une plante, ce qui est contraire aux normes de la CBE.

Le temps passa et les 9-10 mai 2000, l'affaire vint devant la division d'opposition de l'OEB. Des experts firent le voyage des Indes à Munich pour témoigner. D'après un document d'information publié par L. Bullard, l'un d'entre eux, directeur d'une société de biotechnologie, avait développé antérieurement un fongicide analogue que la firme RhônePoulenc aurait considéré comme impossible à breveter et dont il avait envoyé des échantillons à la firme Grace. Quoi qu'il en soit, après les habituelles manœuvres dilatoires, la division d'opposition de l'OEB révoqua le brevet pour manque d'activité inventive. Le 2 avril 2001, le gouvernement des États-Unis et la firme Grace se pourvurent en appel auprès de la chambre de recours technique, en présentant notamment une nouvelle formulation du produit. Le 8 mars 2005 (journée internationale des femmes), les "Trois Grâces " obtinrent gain de cause et la chambre de recours annula irrévocablement le brevet.

Dans les attendus, on lit notamment qu'aucun brevet ne doit être octroyé pour une chose connue au préalable, par exemple dans le cadre de connaissances traditionnelles.

Au-delà de la jurisprudence établie au niveau du brevet européen, des interrogations sont à considérer en l'occurrence. Pourquoi a-t-il fallu une procédure de dix ans pour obtenir une décision dont 
l'évidence s'imposait dès la demande du brevet ? Au-delà des coûts de cette procédure, supportés par les Verts européens et un consortium d'ONG, quelles furent les pertes financières encourues par les utilisateurs des connaissances traditionnelles des graines de margousier aux Indes, suite aux perturbations des marchés locaux par les achats des détenteurs du brevet? Comment de telles pertes peuvent-elles être compensées? Quelles seront les réactions des autres systèmes juridiques, notamment aux États-Unis où des oppositions à des brevets accordés à l'azadirachtine (un produit extrait de margousier qui inhibe la nutrition des insectes) n'ont pas abouti? Ou la situation en Nouvelle-Zélande, où l'antériorité en dehors du pays pour attribuer un brevet n'est pas considérée, encore que des amendements à la législation prévoient la notion de "nouveauté absolue" et la consultation pour avis d'un comité maori lors de l'instruction des demandes de brevets, afin de prendre les valeurs traditionnelles en compte? Quels seront les effets de la révocation du brevet neem sur la jurisprudence de l'OEB?

\section{Conclusion :}

\section{construire la bionomie du $\mathrm{XXI}^{\mathbf{e}}$ siècle}

La "bionomie " qualifie l'administration et la gestion (d'aucuns diront la gouvernance) de la biosphère. Ce terme, qui est en résonance avec des vocables comme agronomie ou économie, identifie une discipline visant à intégrer les interactions entre biosphère et anthroposphère (Semal, 2006). La protection de la propriété intellectuelle (PPI) dans le cadre de la bionomie, présente des aspects à la fois diversifiés et évolutifs, qui vont de la marque (par exemple le pain Poilâne, du nom de son fabricant) jusqu'au biobrevet, en passant par nombre de formes intermédiaires.

Trois accords internationaux majeurs encadrent la PPI en bionomie: les Conventions Trips de 1995, actuellement régies par l'OMC, la Convention sur la biodiversité (CIB) qui a pris cours en
1993, et la Convention Upov dans sa version de 1991. Les conventions "Trips" prescrivent que la PPI des microorganismes (bactéries, levures) est nécessairement couverte par le brevet. Les plantes et les animaux relèvent des États, lesquels peuvent adopter le brevet (comme aux États-Unis et en Europe) ou gérer la PPI via un système sui generis, généralement proche de la convention Upov.

La CIB, à laquelle les États-Unis n'ont pas adhéré, repose sur la reconnaissance de la propriété des États sur leurs ressources génétiques, tout en reconnaissant les contributions des communautés locales et indigènes à la conservation et à l'utilisation durable de la biodiversité.

L'ensemble des systèmes de PPI fonctionne dans un climat de commoditization, terme anglais qui indique que tout objet, qu'il soit matériel ou immatériel, est susceptible d'être acheté ou vendu.

L'évaluation de la biobrevetabilité permet d'identifier trois niveaux :

- celui des micro-organismes utilisés dans les bioindustries, pour lesquels les accords Trips font obligation d'assurer la PPI par le brevet. Ce procédé ne semble pas avoir posé de problème, si ce n'est pour les "génériques " en pharmacie ;

- les cas où les instances responsables de l'application de la PPI accordent des brevets en contrevenant à leurs propres règles, notamment en matière d'inventivité, d'antériorité ou de non-brevetabilité. Ces brevets indus créent de graves perturbations socio-économiques et financières dont la responsabilité incombe à la puissance publique. Quelques balises commencent à émerger qui limitent la brevetabilité du vivant. L'avenir dira si elles feront jurisprudence, afin de contenir les excès en la matière ;

- une zone grise où les effets induits par l'attribution de brevets à des plantes ou animaux perturbent gravement les itinéraires techniques agricoles et les filières de valorisation des productions agroalimentaires. C'est notamment le cas des plantes trangéniques brevetées, dont les impacts négatifs, relevant de "l'infraction innocente" évoquée devant la Cour suprême du Canada, ne sont pas pris en compte.

L'actuel manque de cohérence législative et opérationnelle en matière de breveta- bilité du vivant devrait faire place à une meilleure articulation entre les aspects réalistes (Latour, 2001), éthiques (Kahn, 2000 ; Jacques, 2002), et juridiques (Schmitt, 2002; Chemtob-Concé, 2004). L'heure semble venue de fixer les actes de base de la copropriété, de la covalorisation et de la coresponsabilité qui accompagnent la brevetabilité du vivant. Il s'agit de prendre en compte cette constatation de Percy Schmeiser : "ce sont les gènes de Monsanto, mais ils sont dans mes plantes et sur mes terres".

\section{Références}

Chemtob-Concé M-C. L'approche juridique de la brevetabilité du vivant. Biofutur $2004 ; 245$ 58-60.

Feyt H. La protection de la propriété intellectuelle sur le vivant:historique et débats actuels autour des variétés végétales. OCL 2001a ; 8 : 514-23.

Feyt $\mathrm{H}$. Propriété intellectuelle et sélection dans les pays du Sud. OCL 2001b ; 8 : 546-50.

Feyt $\mathrm{H}$. Le cas des variétés et des ressources génétiques végétales. Biofutur 2004; 246 : 57-60.

Gepts P. Who owns biodiversity and how should the owners be compensated? Plant Physiol $2004 ; 134$ : 1295-307.

Jacques $\mathrm{D}$. La révolution technique. Montréal : éditions du Boreal, 2002.

Kahn A. Et l'homme dans tout ça? Paris : éditions Nil, 2000.

Latour B. L'espoir de Pandore. Paris : éditions La Découverte, 2001.

Pallottini L, Garcia E, Kami G, et al. The genetic anatomy of a patented yellow bean. Crop Sci $2004 ; 44$ : 968-77.

Schmitt P. Le groupe européen d'éthique se penche sur la brevetabilité des biotechnologies. Biofutur $2002 ; 224$ : 42-3.

Semal J. OGM : viol de brevet ou racket légal? L'artichaut $2002 ; 20: 22-8$.

Semal J. Human genome : le reflux. Cah Agric 2005a ; $14: 405$.

Semal J. Biobrevets : la saga du « neem ». Cah Agric 2005b ; $14: 332$.

Semal J. Bio-big-bang. Mons: éditions de l'Échiquier, 2006.

Sulton J. Le génome humain sauvé de la spéculation. Manière de Voir 2004 ; 73 : 88-93.

Supreme Court of Canada. Supreme Court Reports. Ottawa : Supreme Court of Canada, 2004

www.lexum.umontreal.ca/csc-scc/en/rec/html/ 2004scc034.wpd.html.

United States Patent and Trademark Office (USPTO). Office action in ex-parte reexamination (patent 5894079), 04/14/2005. Alexandria (Virginia) : USPTO, 2005. 